\title{
Combined homologous recombination repair deficiency and immune activation analysis for predicting intensified responses of anthracycline, cyclophosphamide and taxane chemotherapy in triple-negative breast cancer
}

Gaoming Liao ${ }^{1 \dagger}$, Zedong Jiang ${ }^{1 \dagger}$, Yiran Yang ${ }^{1 \dagger}$, Cong Zhang ${ }^{2}$, Meiting Jiang ${ }^{3}$, Jiali Zhu', Liwen Xu', Aimin Xie , Min Yan ${ }^{1}$, Yunpeng Zhang ${ }^{1 *}$, Yun Xiao ${ }^{1,4^{*}}$ and Xia Li $i^{1,4^{*}}$

\begin{abstract}
Background: Triple-negative breast cancer (TNBC) is a clinically aggressive disease with abundant variants that cause homologous recombination repair deficiency (HRD). Whether TNBC patients with HRD are sensitive to anthracycline, cyclophosphamide and taxane (ACT), and whether the combination of HRD and tumour immunity can improve the recognition of ACT responders are still unknown.

Methods: Data from 83 TNBC patients in The Cancer Genome Atlas (TCGA) was used as a discovery cohort to analyse the association between HRD and ACT chemotherapy benefits. The combined effects of HRD and immune activation on ACT chemotherapy were explored at both the genome and the transcriptome levels. Independent cohorts from the Molecular Taxonomy of Breast Cancer International Consortium (METABRIC) and Gene Expression Omnibus (GEO) were adopted to validate our findings.
\end{abstract}

\footnotetext{
*Correspondence: zhangyp@hrbmu.edu.cn; xiaoyun@ems.hrbmu.edu.cn; lixia@hrbmu.edu.cn

${ }^{\dagger}$ Gaoming Liao, Zedong Jiang and Yiran Yang contributed equally to this work as first authors.

${ }^{1}$ College of Bioinformatics Science and Technology, Harbin Medical University, Harbin 150081, Heilongjiang, China

Full list of author information is available at the end of the article
}

C C The Author(s). 2021 Open Access This article is licensed under a Creative Commons Attribution 4.0 International License, which permits use, sharing, adaptation, distribution and reproduction in any medium or format, as long as you give appropriate credit to the original author(s) and the source, provide a link to the Creative Commons licence, and indicate if changes were made. The images or other third party material in this article are included in the article's Creative Commons licence, unless indicated otherwise in a credit line to the material. If material is not included in the article's Creative Commons licence and your intended use is not permitted by statutory regulation or exceeds the permitted use, you will need to obtain permission directly from the copyright holder. To view a copy of this licence, visit http://creativecommons.org/licenses/by/4.0/ The Creative Commons Public Domain Dedication waiver (http://creativecommons.org/publicdomain/zero/1.0/) applies to the data made available in this article, unless otherwise stated in a credit line to the data. 
Results: HRD was associated with a longer ACT chemotherapy failure-free interval (FFI) with a hazard ratio of 0.16 $(P=0.004)$ and improved patient prognosis $(P=0.0063)$. By analysing both HRD status and ACT response, we identified patients with a distinct TNBC subtype (ACT-S\&HR-P) that showed higher tumour lymphocyte infiltration, IFN- $\gamma$ activity and NK cell levels. Patients with ACT-S\&HR-P had significantly elevated immune inhibitor levels and presented immune activation associated with the increased activities of both innate immune cells and adaptive immune cells, which suggested treatment with immune checkpoint blockade as an option for this subtype. Our analysis revealed that the combination of HRD and immune activation enhanced the efficiency of identifying responders to $A C T$ chemotherapy ( $A \cup C=0.91, P=1.06 \mathrm{e}-04$ ) and synergistically contributed to the clinical benefits of TNBC patients. A transcriptional HRD signature of ACT response-related prognostic factors was identified and independently validated to be significantly associated with improved survival in the GEO cohort $(P=0.0038)$ and the METABRIC dataset $(P<0.0001)$.

Conclusions: These findings highlight that HR deficiency prolongs FFI and predicts intensified responses in TNBC patients by combining HRD and immune activation, which provides a molecular basis for identifying ACT responders.

Keywords: Triple-negative breast cancer, ACT chemotherapy, Homologous recombination repair deficiency, Failurefree interval, Immune checkpoint

\section{Background}

Triple-negative breast cancer (TNBC) characterized by absent or minimal expression of oestrogen receptors (ER), progesterone receptors (PR) and human epidermal growth factor receptor 2 (HER2) is a highly heterogeneous and aggressive disease and has the worst prognosis among the different subtypes of breast cancer $[1,2]$. Among the chemotherapy regimens used for TNBC, sequential anthracycline (A) and cyclophosphamide (C) followed by taxane (T) (ACT) are some of the preferred regimens in international guidelines [3, 4]. However, approximately $30-40 \%$ of patients with residual disease (RD) after surgery treated with ACT-based therapy will develop metastatic disease and death [5].

The detailed molecular characterization of refractory tumours is a prerequisite to understanding therapy resistance and developing reasonable treatment strategies. Homologous recombination repair (HRR) is a highfidelity repair mechanism specifically for DNA doublestrand breaks (DSBs) [6]. BRCA1/2 are key components in the HR-mediated DNA DSB repair mechanism, and mutations in BRCA1/2 are typical molecular alterations that lead to homologous recombination repair deficiency (HRD) and sensitivity to DNA damage agents [7, 8]. In vitro and preclinical studies have shown that tumours with HR deficiency are sensitive to platinum-containing and/or DNA damage mutagens, which significantly increases the patient response rate and prolongs survival $[9,10]$. However, whether HR deficiency could improve the response to DNA-damaging or repair-inhibiting therapies such as doxorubicin (which induces DNA DSBs) and cyclophosphamide (an alkylating agent that causes DNA crosslinks that lead to DSBs) $[8,10]$ in TNBCs remains poorly characterized, although BRCA1/
2 germline mutations have been shown to promote the pathological complete response (pCR) in early TNBC patients who received ACT chemotherapy [11].

Among chemotherapy options in TNBC, combining immunomodulatory therapy (such as atezolizumab) with nab-paclitaxel and anthracycline-based chemotherapy is potentially advantageous, significantly improving pCR rates with an acceptable safety profile $[12,13]$. Shibata et al. showed that the DNA DSB repair pathway upregulated the $P D-L 1$ expression in cancer cells by activating STAT1 and STAT3 signalling and the IRF1 pathway [14]. Unrepaired DSBs regulate the tumour immune microenvironment through a series of molecular and cellular mechanisms, such as increasing genomic instability, activating immune pathway activation and facilitating $P D-L 1$ expression on cancer cells, which might promote responsiveness to immune checkpoint inhibitors (ICIs) $[15,16]$. The clinical and translational data indicated that low-dose chemotherapy may be utilized to stimulate anticancer immune responses. For example, short-term doxorubicin treatment may promote a more favourable tumour microenvironment and increase the likelihood of a response to $P D-1$ blockade in TNBC [17]. Additionally, low-dose cyclophosphamide induced antitumour $\mathrm{T}$ cell responses in metastatic colorectal cancer [18]. These studies indicated that it is necessary to consider the mechanism of the tumour immune microenvironment to characterize the effect of HRD on the ACT chemotherapy response.

Here, we performed an integrated genome analysis of TNBC patients who received ACT treatment after tumour resection, specifically focusing on HRD and the immune microenvironment and their combined effect on the treatment response and clinical outcomes. A 
transcriptional HRD signature as a prognostic factor related to ACT chemotherapy was identified and independently validated to be significantly associated with improved survival in the GEO cohorts and the METABRIC dataset. Our goal is to evaluate the combination of HRD and immune activation as a potentially stronger tool to predict which TNBC patients might achieve a valuable response to ACT-based preoperative chemotherapy.

\section{Methods}

\section{Sample collection and datasets}

\section{Discovery cohort}

In this study, we downloaded whole-exome sequencing (WES) data and gene expression profiles (RNA-Seq, RSEM standardization) for breast cancer patients in The Cancer Genome Atlas (TCGA) from cBioPortal [19]. Additionally, the patient survival and clinical phenotype (including age, PAM50 subtype and AJCC tumour stage) data were obtained. The sampling time points of patients and medication information related to the anticancer drugs used (including start/end time points of treatment) were obtained from the Genomic Data Commons Data Portal (GDC Data Portal). We reviewed the drug names and manually standardized them through
DrugBank [20]. According to the dates given for sampling and first treatment, we obtained a clear cohort of primary breast cancer patients who received ACT treatments after tumour resection (Fig. 1). After determining the immunohistochemistry (IHC) status of ER, PR and HER2 and selecting patients for whom exome sequencing data were available, we established a final cohort of 83 TNBC patients (Table 1). Patients who were sensitive to ACT treatment were defined as having complete responses to ACT or a failure-free interval (FFI) above the median. Patients with disease progression or no improvement after ACT treatment ended (progressive disease/stable disease), or an FFI below the median were defined as resistant to ACT (Table 1).

\section{Validation cohorts}

We downloaded data from GEO (GSE25055, GSE25065 and GSE41998), including expression profile, ACT response and clinical phenotype, for validation cohorts of TNBC patients who received neoadjuvant ACT therapy after sample procurement [21, 22]. A cohort of 299 TNBC patients who received ACT chemotherapy after tissue sample collection was obtained from METABRIC [23]. In addition, two cohorts of TNBC patients (Hess2006, neoadjuvant therapy; Chin2006, adjuvant

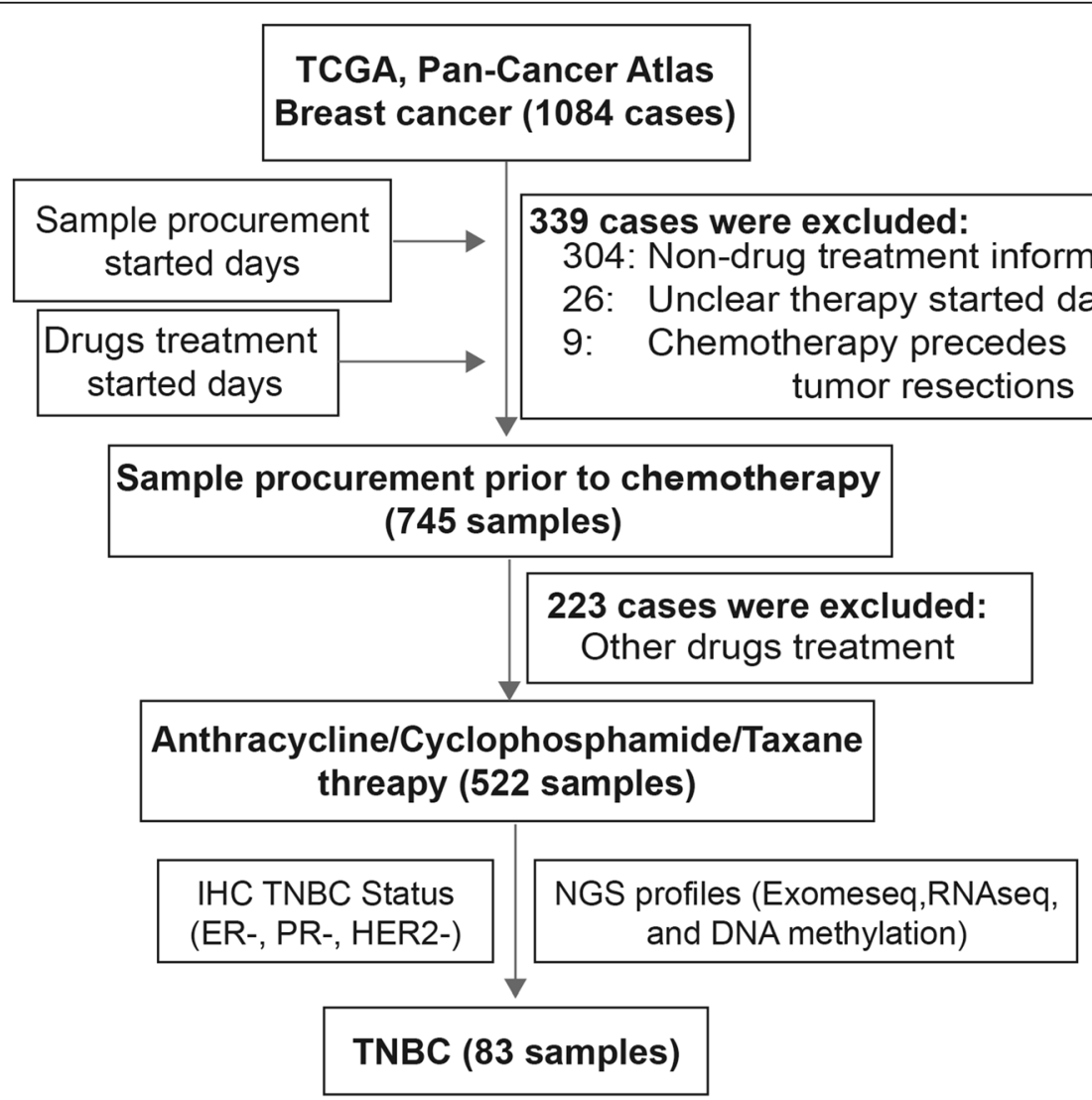

Fig. 1 Sampling procedure for TNBC patients prior to ACT chemotherapy 
Table 1 Patient and tumour characteristics of TNBC

\begin{tabular}{ll}
\hline Baseline characteristics $(\boldsymbol{n}=\mathbf{8 3})$ & $\boldsymbol{N}$ (\% or range) \\
\hline Age (mean) & $52(29-78)$ \\
AJCC stage & $18(21.7)$ \\
Stage I, stage IA & $52(62.7)$ \\
Stage IIA, stage IIB & $12(14.5)$ \\
Stage IIIA, stage IIIB, stage IIIC & \\
PAM50 subtype & $64(80.7)$ \\
Basal & $5(6.0)$ \\
Her2 & $1(1.2)$ \\
LumB & \\
HRD status & $48(57.8)$ \\
HR deficiency & $35(42.2)$ \\
HR proficiency & \\
BRCA1/2 mutation & $6(7.2)$ \\
BRCA1 & $5(6.0)$ \\
BRCA2 & $7(7.2)$ \\
BRCA1 promoter methylation & \\
ACT chemotherapy response & $21(25.3)$ \\
Complete response & $2(2.4)$ \\
Partial response & $1(1.2)$ \\
Progressive disease & $59(71.1)$ \\
- & \\
ACT response status & $39(47.0)$ \\
Rensitive & $44(53.0)$ \\
\hline &
\end{tabular}

therapies) who were treated with ACT after sample procurement were acquired from UCSC Xena [24, 25]. Hess2006 cohort patients undergoing diagnostic biopsy were prior to ACT neoadjuvant therapy. Information on all TNBC patients used in this study is provided in Additional file 1: Table S1.

\section{HRD score, mutational signature and HRDetect score}

The HRD score was calculated based on the number of specific lesions in the genome, including loss-ofheterozygosity $(\mathrm{LOH})$, large-scale transitions (LST) and the number of telomeric allelic imbalances (ntAI). Following Thorsson et al.'s research on TCGA data [26], we extracted the HRD scores of TNBC patients. Somatic point mutational signatures were determined with the deconstructSigs $\mathrm{R}$ package [27] by using the COSMIC signatures as a mutational-process matrix. The WESbased HRDetect scores were calculated using the lasso logistic model by fitting multiple predictors related to HRD, including the HRD score, the contribution of major mutational signatures in breast cancers (such as signature 1, signature 3, signature 6 and signature 20) and the insertion/deletion ratio [28]. The weights of the whole exome-specific model were trained on 560 artificial whole exomes [29].

\section{BRCA1 promoter methylation analysis}

We acquired DNA methylation data (Illumina Human Methylation 450) from the UCSC Xena database. The transcription start site (TSS) information of the human reference genome (GRCh38) was obtained from Ensembl. The promoter region was defined as the 1500 bp upstream and $500 \mathrm{bp}$ downstream of the TSS. We identified a robust probe $(\operatorname{cg} 13782816)$ for the BRCA1 gene located in the promoter region through the gene symbol annotation information. BRCA1 promoter methylation (epigenetic silencing) was defined as a cg13782816 methylation level exceeding 0.9 .

Immune microenvironment mechanism of TNBC patients The $\mathrm{R}$ package CIBERSORT [30] was used to calculate the level of immune cell infiltration in TNBC patients. The IFN- $\gamma$ score of the TNBC patients was calculated using the Tumor Immune Dysfunction and Exclusion (TIDE) online analysis tool [31]. The immune molecular and cellular characteristics of TCGA BRCA patients were obtained from a previous study [26], including tumour lymphocyte infiltration (TLI) score, regulatory macrophages (Mregs), TCR/BCR Shannon, TCR/BCR richness and TGF- $\beta$ response and neoantigens. Tumour mutation burden (TMB) was defined as the total number of nonsynonymous single nucleotide and indel variants.

\section{Gene set enrichment analysis and pathway activity calculation}

We downloaded all the pathways of Collection 2 (C2) and their included gene sets from MSigDB (v7.2) [32]. Patients with the ACT-S\&HR-P subtype served as the case group, other patients served as the control group and the $\mathrm{R}$ package DESeq2 was used for differential expression analysis. Gene set enrichment analysis (GSEA) was performed using the $\mathrm{R}$ package clusterProfiler. We acquired the gene sets of both immune cell types and core biological pathways from previous studies [33, 34] and calculated the activity of these pathways/immune cells utilizing the R package GSVA. We obtained sets of immunostimulators and immune inhibitors based on known studies [35, 36] and explored the differences in the expression of these genes between patients with ACT-S\&HR-P and other TNBCs.

\section{Immune score of TNBC patients}

Two different methods were used to calculate the immune score (IS) of each TNBC patient based on the gene expression levels. (1) Due to the significantly higher activities of immune response-related pathways (including interferon and immune checkpoint blockade-related 
pathways, as well as CD8 effector T cells) in the ACTS\&HR-P subtype, we calculated the IS according to the sum of the activities of these pathways (Additional file 1: Table S2). (2). The IS was also calculated using the average expression of prognostic immune markers in breast cancer [37].

\section{Identification of the HRD expression signature and computation of the HRD-related prognostic score}

After grouping TNBC samples (HR deficiency and HR proficiency) according to HRD status and performing differential expression analysis using the $\mathrm{R}$ package DESeq2, we identified differentially expressed genes (DEGs) using FDR $\leq 0.05$ and fold change $\geq 2$ or $\leq 1 / 2$ as the threshold. Univariate Cox regression analysis was performed in the FFI of TNBC patients based on the expression levels of all DEGs. Finally, 15 HRD-related prognostic factors (HRD expression signature) were determined after removing redundant factors using lasso logistic regression. Among those signature genes, 4 were strongly overexpressed (FDR $\leq 0.05$, fold change $\geq 2$ ), and 11 were downregulated (FDR $\leq 0.05$, fold change $\leq$ 1/2) in HR-deficient compared to HR-proficient cases.

Considering the transcription levels of the HRD expression signature and the hazard ratio calculated by Cox regression analysis, the prognostic score (PS) of upregulated factors (all hazard ratio $<1$, protective factor) and downregulated factors (all hazard ratio $>1$, risk factor) were computed as follows:

$$
\mathrm{PS}_{i}=\sum_{j=1}^{n} \frac{\operatorname{Exp}_{i j}}{\mathrm{HR}_{j}}
$$

where $\mathrm{HR}_{j}$ represents the hazard ratio of upregulated (or downregulated) factor $j$ in the Cox model. Exp $i j$ represents the expression levels (log-transformed) of upregulated (or downregulated) factor $j$ in sample $i$. We calculated the HRD-related prognostic score (HRDPS) of patients based on the PS of both upregulated and downregulated factors as follows:

$$
\mathrm{HRDPS}_{i}=\mathrm{PS}_{i, \text { up }}-\mathrm{PS}_{i, \text { down }}
$$

where $\mathrm{PS}_{i \text {, up }}$ represents the prognostic score of upregulated factors in sample $i$, and $\mathrm{PS}_{i \text {, down }}$ represents the prognostic score of downregulated factors in sample $i$.

\section{Statistical analyses}

$\mathrm{R}$ Project (version 4.02) for statistical computing was used in this study. The nonparametric Wilcoxon ranksum test was used to explore the statistical significance between discrete variables (such as HRD status and $\mathrm{ACT}$ response status) and continuous indicators (such as immune cell infiltration and pathway activities). The nonparametric Kruskal-Wallis test was used for comparisons among multiple groups, with Benjamini and Hochberg false discovery rate (FDR) correction. For some comparisons (activated NK cells, M0 macrophages and activated mast cells), we additionally performed a combinatorial method that Wilcoxon's rank-sum test with continuity correction combined 10,000 iterations. Fisher's exact test was used to examine the relationships between HRD status and HRR-related gene mutations, $B R C A 1$ promoter methylation and the effect on the response to ACT chemotherapy. Clinical outcomes and FFI were compared using the log-rank test. KaplanMeier graphs were plotted using standard methodologies. A Cox proportional hazards model was used to calculate the hazard ratios and corresponding 95\% confidence intervals (CIs) with adjustments for age, tumour stage and disease stage. Statistical significance was set at two-tailed $P<0.05$.

\section{Results}

Genomic alterations and homologous recombination repair defects were widespread in TNBC patients

We acquired data for 83 TNBC patients who underwent ACT chemotherapy after tissue sample collection based on the dates given for sampling and first treatment (Fig. 1). Consistent with a previous study [38], most TNBC patients belonged to the basal subtype (88\%; Fig. 2A). As expected, TNBC patients carrying mutations in TP53 (84\%), PTEN (11\%) and BRCA1/2 (8\% and 7\%, respectively) were relatively frequent among breast cancer (BC) patients in the PanCancer Atlas [39] (Fig. 2A, Additional file 1: Fig. S1A). In contrast, the oncogene PIK3CA $(11 \%)$ was less prone to the mutation in TNBCs than all BC patients.

Biomarkers for homologous recombination repair deficiency in cancers have attracted great interest from researchers $[8,40]$. HR deficiency was defined as either a deleterious tumour BRCA1/2 (tBRCA) mutation or a predefined HRD score $\geq 42$ [8], which was determined in $57.8 \%(48 / 83)$ of TNBC patients (Fig. 2A, Table 1). Our results showed that most TNBC patients with BRCA1/2 mutations presented HRD scores ${ }^{\text {high }}(\geq 42)$ and dominant mutational signature 3 (SBS3) activity ( $\geq$ 0.3) (both $8 / 11$; Fig. 2B). Indeed, the patients with $\mathrm{HR}$ deficiency showed significantly higher SBS3 exposure than patients with HR proficiency $(P=4.3 \mathrm{e}-04$, Wilcoxon rank-sum test; Fig. $2 C$ ). In addition to $B R C A 1 / 2$, several important components of the HRR pathway, such as RAD54 family members (including $R A D 54 B$ and $R A D 54 L$ ), DNA polymerase members (including POLD1, $P O L H$ and $P O L Q)$ and PARP1, PALB2 and TP53BP1, occurred mainly in the HR deficient samples $(29 / 48, P=$ 3.04e-04, Fisher's exact test; Fig. 2D, Additional file 1: Fig. S1B). Consistent with previous studies, we also found that HR-deficient patients showed a higher 


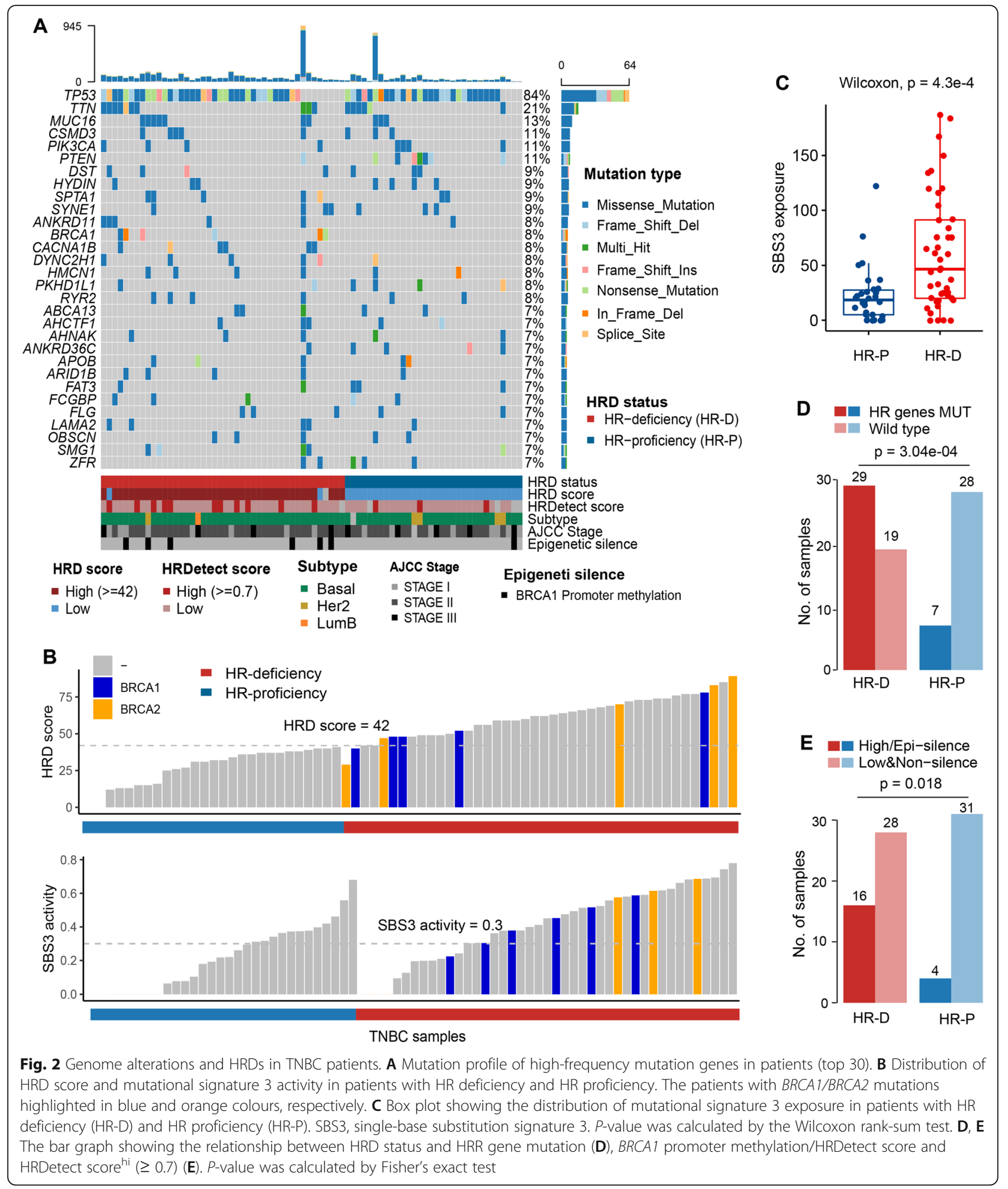

proportion of $B R C A 1$ promoter methylation and HRDetect score ${ }^{\text {high }}(16 / 48, P=0.018$, Fisher's exact test; Figs. $2 \mathrm{~A}, \mathrm{E})[28,41]$. These results indicated that HRD could be characterized by mutations in HRR-related genes, SBS3 exposure and BRCA1 promoter hypermethylation.
Homologous recombination repair deficiency correlates with ACT chemotherapy benefits

Accumulated evidence has shown that HRD is associated with a better prognosis for patients with a variety of solid tumours [42, 43]. Whether a better prognosis is related to 

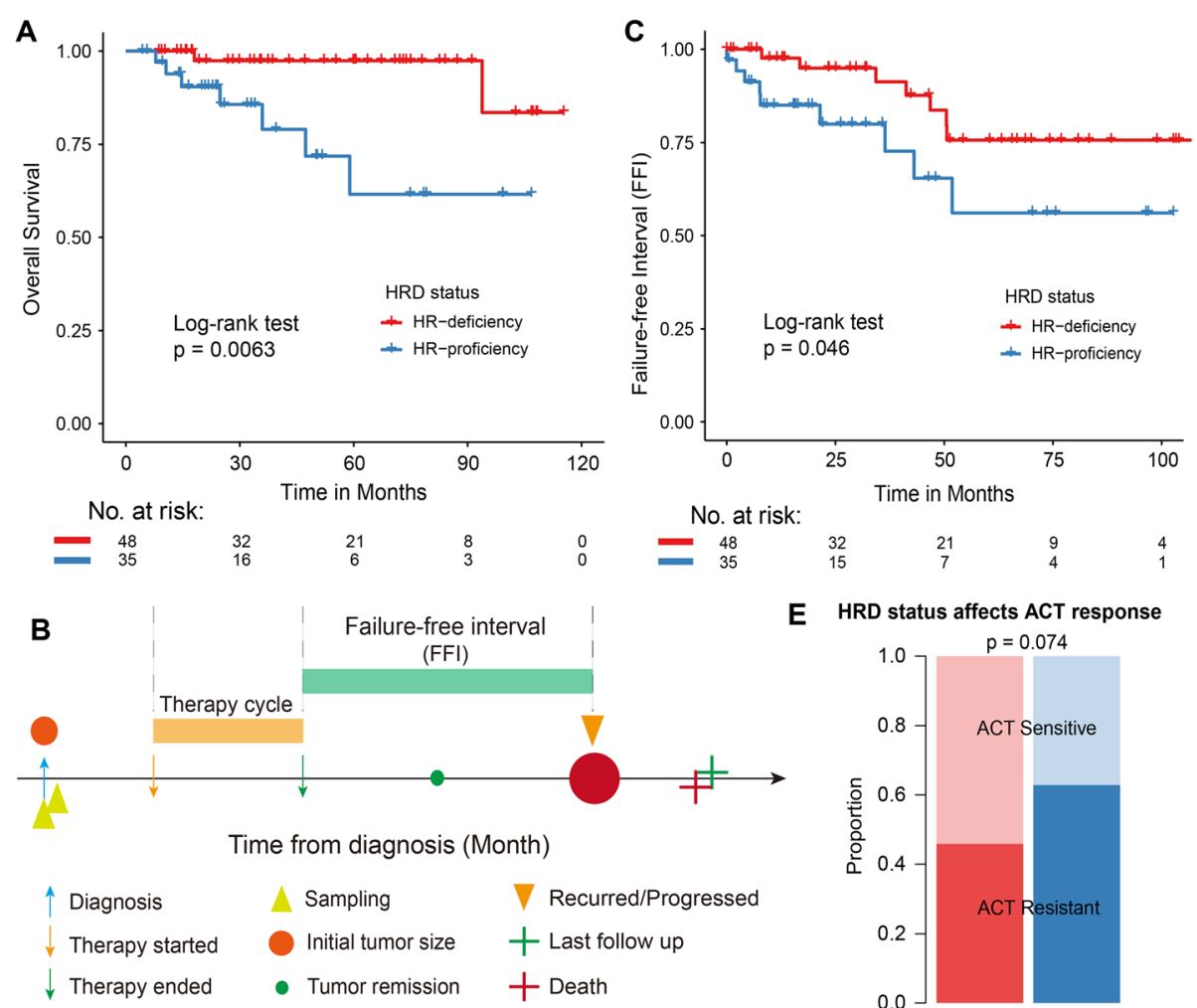

E HRD status affects ACT response

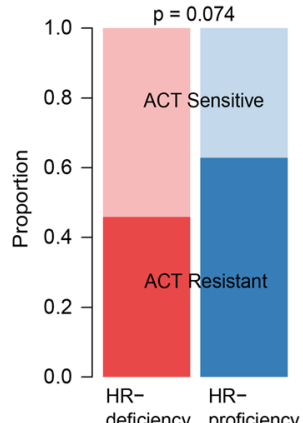

\begin{tabular}{lcc} 
D & $\begin{array}{c}\text { No. of } \\
\text { Patients }\end{array}$ & $\begin{array}{c}\text { Multivariate Cox }(\mathrm{FFI}) \\
\text { Hazard ratio }(95 \% \mathrm{Cl})\end{array}$ \\
\hline HRD & $(\mathrm{N}=83)$ & $(0.044-16-0.55)$
\end{tabular}

deficiency proficiency

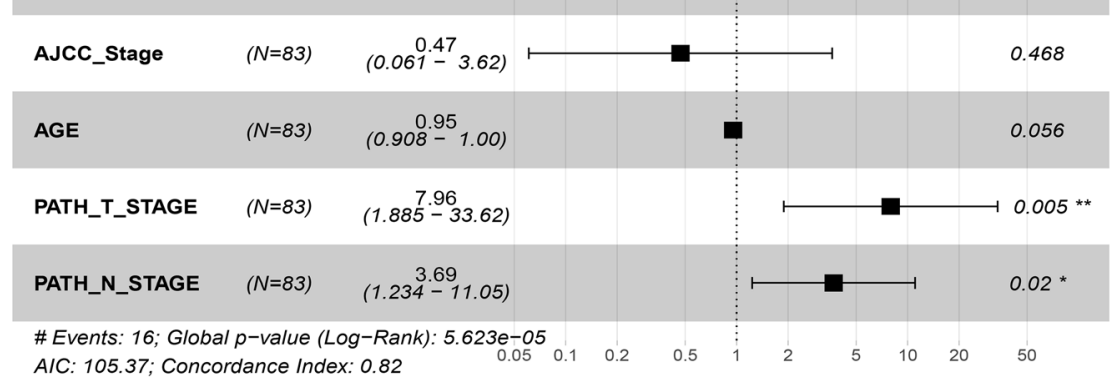

Fig. 3 Homologous recombination repair deficiency correlates with ACT chemotherapy benefits. A, C Kaplan-Meier graphs of HRD status on OS (A) and FFI (C). Statistical significance was calculated using the log-rank test. B Conceptual depiction of the TNBC patients undergoing ACT chemotherapy. D Forest plot illustrating the HR $(95 \% \mathrm{Cl})$ for FFI calculated using the multivariate Cox proportional hazard models. HR, hazard ratios; $\mathrm{Cl}$, confidence interval. E Distribution of ACT sensitive (light colour) or resistant (dark colour) in patients with HR deficiency (red) and HR proficiency (blue)

HRD in TNBC patients who received ACT chemotherapy has not been well characterized. Our results revealed that compared with patients with HR proficiency, patients with HR deficiency showed significantly better overall survival (OS; $P=0.0063$, log-rank test; Fig. 3A) and diseasespecific survival (DSS; $P=0.023$, log-rank test; Additional file 1: Fig. S2A) after treatment with ACT. Specifically, the 5 -year OS rate for HR-deficient patients was $98 \%$, while that for HR-proficient patients, it was only $61 \%$ (Fig. 3A). Multivariate Cox regression analysis showed that HR deficiency was an independent protective factor associated with prolonged patient OS $(P=0.002, \log$-rank test; Additional file 1: Fig. S2B $)$ and DSS $(P=0.014$, log-rank test; Additional file 1: Fig. S2C) in TNBC after adjusting for clinical factors including age, AJCC stage and TNM stage.

Furthermore, to explore whether HR deficiency can indeed benefit TNBC patients' response to ACT treatment, we determined the ACT chemotherapy failure-free interval (FFI) of patients based on the period from the end of treatment to tumour progression/recurrence or death 


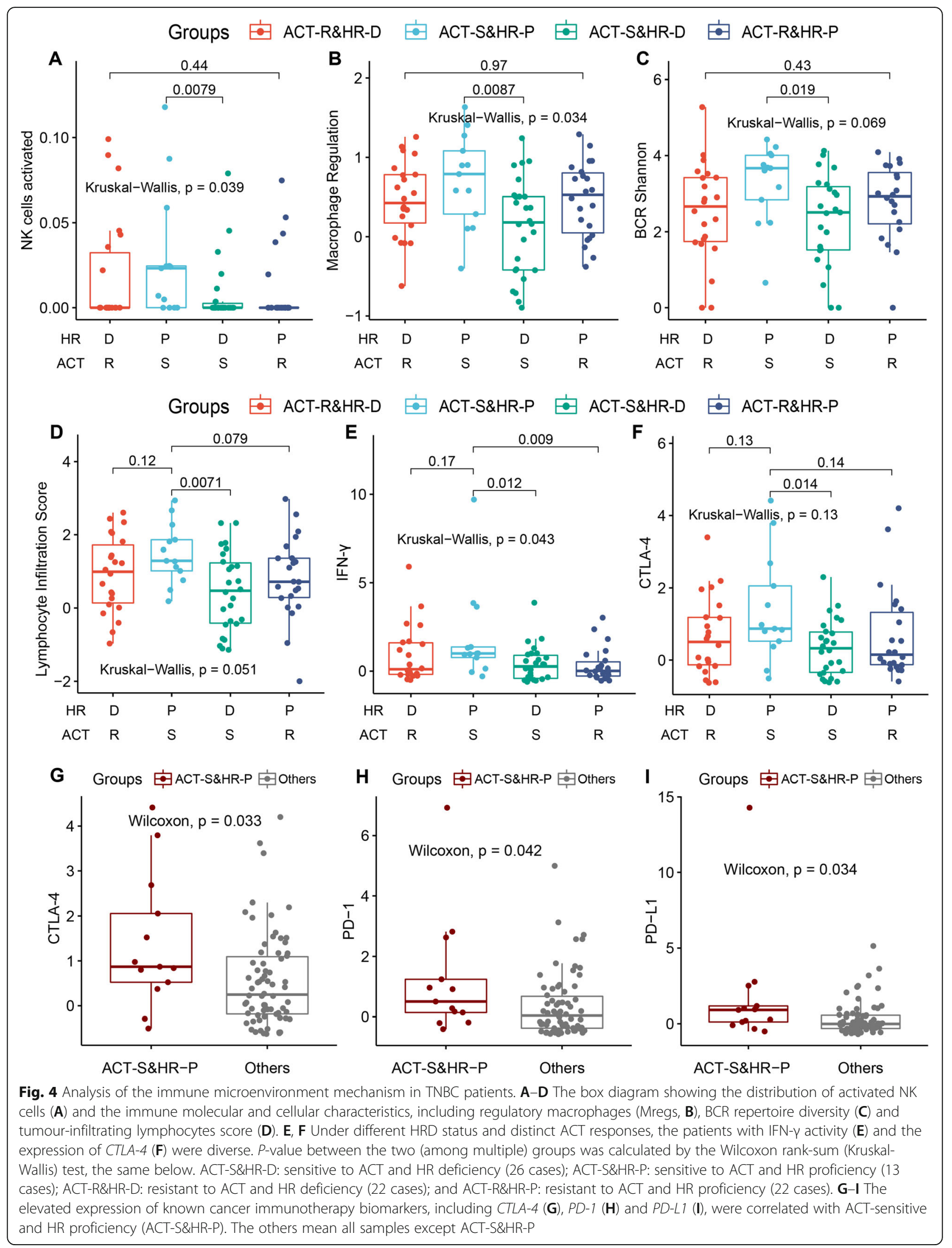


(Fig. 3B). Our results showed that HR deficiency was correlated with durable response to ACT chemotherapy $(P=$ 0.046, log-rank test; Fig. 3C). The 5-year FFI rate for HRdeficient patients was $75 \%$, while that for HR proficiency, it was only $52 \%$. Cox regression analysis showed that HR deficiency was a significant protective factor for FFI in TNBC patients with a hazard ratio of 0.16 (95\% CI $0.044-$ $0.55, P=0.004$; Fig. 3D), which improved the response interval to ACT chemotherapy. In addition, we found that HR-deficient patients tended to be more sensitive to ACT chemotherapy (54.2\% for sensitive, $37.1 \%$ for resistant), compared with HR-proficient patients (37.1\% for sensitive, $62.9 \%$ for resistant; $P=0.074$, Fisher's exact test; Fig. 3E).

\section{Revealing the diversity of the immune microenvironment} related to ACT responses utilizing HRD status

Unrepaired DNA damage, especially HRD, modulates the tumour immune microenvironment through a range of molecular and cellular mechanisms $[15,16]$. Lowdose doxorubicin and cyclophosphamide chemotherapy may stimulate anticancer immune responses and promote a more favourable tumour microenvironment [17, 18]. We speculated that the impact of HRD on the immune microenvironment was related to the ACT response in TNBC patients. As expected, we found that the effect of HRD on immune cell infiltration showed significant differences in distinct ACT response groups (Fig. 4, Additional file 1: Fig. S3). For example, in the ACT-sensitive (ACT-S) group, NK cells showed higher infiltration in HR-proficient (HR-P) samples $(P=0.0079$, Wilcoxon rank-sum test, same below; Fig. 4A). In contrast, M0 macrophages and mast cells presented higher infiltration levels in HR-deficient (HR-D) samples ( $P$ values were 0.02 and 0.012 , respectively; Additional file 1: Fig. S3AB). Interestingly, differences in these immune cells were not observed in the ACT-resistant (ACT-R) group (Fig. 4A, Additional file 1: Fig. S3AB). Additionally, in the ACT-sensitive group, we found that regulatory macrophages (Mregs) were significantly activated in HR-proficient patients $(P=0.0087$, Wilcoxon rank-sum test; Fig. $4 \mathrm{~B})$. These patients showed T cell/B cell receptor $(\mathrm{TCR} / \mathrm{BCR})$ repertoire diversity ( $P$-values of 0.041 and 0.019, respectively; Fig. 4C, Additional file 1: Fig. $\mathrm{S} 3 \mathrm{C}$ ) and TCR/BCR richness ( $P$-values of 0.036 and 0.018 , respectively; Additional file 1: Fig. S3DE). Diversified TCR/BCR receptors are the basic attributes of an effective immune system, allowing $\mathrm{T} / \mathrm{B}$ cells to target multiple types of endogenous or exogenous antigens [44, 45]. However, we did not find corresponding results in the ACT-resistant patient group (Fig. 4C, Additional file 1: Fig. S3CDE).

Studies have suggested that neoantigens presentation in tumours promotes the release of IFN- $\gamma$ from tumourinfiltrating lymphocytes (TILs), and the released IFN- $\gamma$ upregulates $P D-L 1$ expression in immune cells and tumours [46, 47]. We found that in the ACT-sensitive group, HR-proficient samples showed higher TIL infiltration $(P=0.0071$, Wilcoxon rank-sum test, same below) and IFN- $\gamma$ activity $(P=0.012)$ compared to HRdeficient samples (Fig. 4D, E). Among HR-proficient patients, those sensitive to ACT also showed higher TIL scores $(P=0.079$, Wilcoxon rank-sum test, same below) and stronger IFN- $\gamma$ activity $(P=0.009)$ than ACTresistant patients (Fig. 4D, E). The patients with ACTS\&HR-P (sensitive to ACT and HR proficiency) were associated with higher IFN- $\gamma$ activity $(P=0.011$, Wilcoxon rank-sum test; Additional file 1: Fig. S4A) and lower TGF beta response (Additional file 1: Fig. S4B), implying an intense immune response in this subtype. Furthermore, for the known cancer immunotherapy biomarkers, we found that their expression levels were correlated with ACT response and HRD status (Fig. 4F, Additional file 1: Fig. S4CD). For example, significantly higher expression levels of $C T L A-4, P D-1$ and $P D-L 1$ were found in ACT-S\&HR-P patients $(P<0.05$, Wilcoxon rank-sum test; Fig. 4G-I). In particular, patients with HR deficiency showed higher TMB $(P=0.0031$, Wilcoxon ranksum test; Additional file 1: Fig. S4E) and neoantigen levels $(P=0.0018$, Wilcoxon rank-sum test; Additional file 1: Fig. S4F), which may be related to the fact that HRD exacerbates DNA DSBs, thereby promoting genome instability and causing the release of molecular antigens [48].

\section{Immune checkpoint blockade as an optional treatment for patients with ACT-S\&HR-P subtype}

The clinical and translational data indicated that shortterm doxorubicin treatment may increase the likelihood of a response to $P D-1$ blockade in TNBC [17]. Considering the results of our study, we postulated that ACTS\&HR-P patients may benefit from immune checkpoint blockade (ICB) therapy. To test this postulate, we performed GSEA on the C2 pathways from MSigDB (v7.2) (the "Methods" section). We found that the genes that were upregulated in ACT-S\&HR-P patients were enriched in multiple immune response-related pathways, such as interferon-gamma signalling $(\mathrm{NES}=2.53$, FDR < 0.001; Fig. 5A, Additional file 1: Fig. S6A), interferon signalling $(\mathrm{NES}=2.30, \mathrm{FDR}<0.001$; Additional file 1 : Fig. $\mathrm{S} 5 \mathrm{~A})$ and type II interferon signalling IFN- $\gamma(\mathrm{NES}=$ 2.36, FDR < 0.001; Additional file 1: Fig. S5B). In addition, ICB-related pathways, including cancer immunotherapy by $P D-L$ blockade $(\mathrm{NES}=2.41$, FDR < $0.001)$, the $C T L A-4$ pathway $(\mathrm{NES}=2.55, \mathrm{FDR}<0.001)$ and $C D 28$ family costimulation $(\mathrm{NES}=2.45, \mathrm{FDR}<$ 0.001 ), were related to the upregulated genes in this subtype (Fig. 5B, Additional file 1: Fig. S5CD). In particular, natural killer cell-mediated cytotoxicity $(\mathrm{NES}=2.05$, 

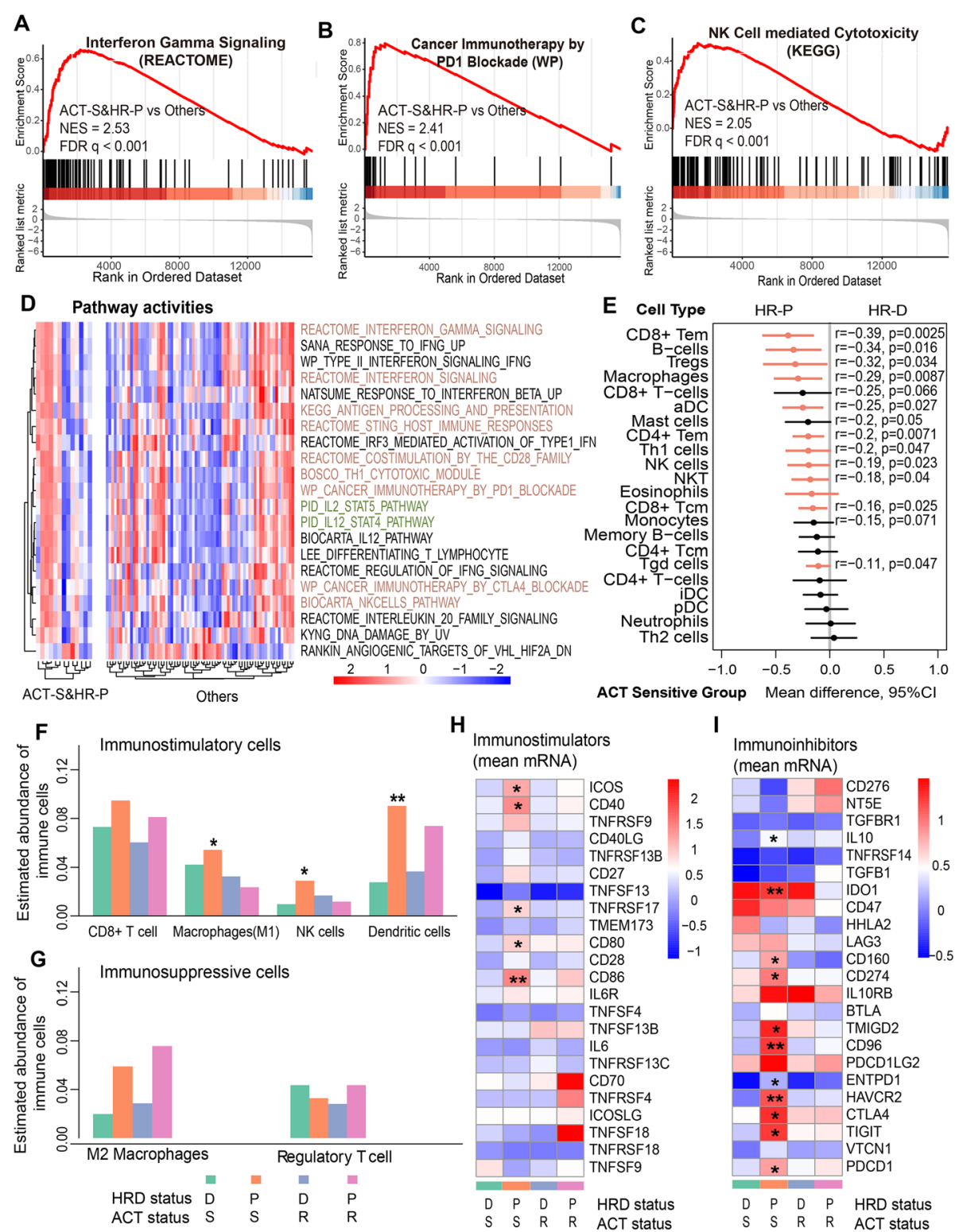

Fig. 5. Potential application of immune checkpoint blockade in the ACT-S\&HR-P subtype. A-C Representative gene set enrichment analysis plot showing upregulated interferon gamma signalling (A), cancer immunotherapy by PD-L blockade (B) and natural killer cell-mediated cytotoxicity (C) in the ACT-S\&HR-P (sensitive to ACT and HR proficiency) subtype versus the other subtypes. NES, normalized enrichment score. D Pathway activity in ACT-S\&HR-P patients and other patients. The highlighted pathways are indicated as being focused on in this research. $\mathbf{E}$ In the ACT-sensitive group, immune cell activity scores were higher in the HR-proficient samples. The dots depict the mean difference of immune cell activity scores in HRdeficient samples compared to HR-proficient samples, and the lines show the $95 \%$ confidence interval (Cl) for the difference. $P<0.05$ was considered significant (red colour), Wilcoxon rank-sum test. See Figure $\mathbf{S} 6$ for the full name of immune cell types. F, G Relative number of immune-stimulatory cells $(\mathbf{F})$ and immune-suppressive cells $(\mathbf{G})$ in the four TNBC subtypes calculated using the CIBERSORT algorithm. $\mathbf{H}, \mathbf{I}$ The expression of immune stimulatory molecules $(\mathbf{H})$ and immune checkpoint genes (I) in four TNBC subtypes. ${ }^{* *} P<0.001,{ }^{* *} P<0.01,{ }^{*} P<0.05$

FDR $<0.001$; Fig. 5C) and antigen processing and presentation $(\mathrm{NES}=2.63$, FDR $<0.001$; Additional file 1: Fig. $\mathrm{S} 5 \mathrm{E})$ were also enriched to the upregulated genes in this subtype.

Consistent results were found using pathway activity. For example, cancer immunotherapy by CTLA-4/PD-1 blockade was activated in ACT-S\&HR-P patients (mean differences $>0, P<0.05$, Wilcoxon rank-sum test, the same below; Fig. 5D, Additional file 1: Fig. S6B). Similarly, the immune response-related pathways mentioned above also showed significant activation in this subtype, including interferon-gamma signalling (mean difference $=0.29, P=0.0078$ ), NK cell pathway (mean difference $=$ $0.31, P=0.011)$ and antigen processing and presentation 
(mean difference $=0.28, P=0.0035$ ) (Fig. 5D, Additional file 1: Fig. S6B). The JAK-STAT signalling pathway plays critical roles in the coordination of the immune system, especially for cytokine receptors, and it can regulate the polarization of helper $\mathrm{T}$ cells [14]. Our results showed that the genes upregulated in ACT-S\&HR-P patients were enriched to the JAK-STAT signalling pathway (NES $=2.58$, FDR < 0.001; Additional file 1: Fig. S5F). Additionally, the IL2-STAT4 pathway (NES $=2.62$, FDR $<0.001)$ and IL2STAT5 pathway $(\mathrm{NES}=2.25, \mathrm{FDR}<0.001)$ showed correlations with the upregulated genes in this subtype (Additional file 1: Fig. S6A). Additionally, the pathways related to the inflammatory response, including the IL2-STAT4/5 pathways presented higher activities in this subtype (Fig. 5D, Additional file 1: Fig. S6B). These results suggested that ACT-S\&HR-P patients exhibit surprisingly elevated immune activities by activating the immune response pathways.

By analysing the types of immune cells [33], we found that among HR-proficient patients, both innate immune cells (such as activated DCs [aDCs], mast cells, macrophages and natural killer (T) cells [NKs/MKTs]) and adaptive immune cells (such as $\mathrm{T}$ helper 1 [Th1], CD8+ $\mathrm{T}$ central memory [Tcm], CD8+ T effector memory [Tem] and CD4+ Tem cells) were activated only in the ACT-sensitive group $(P<$ 0.05 , Wilcoxon rank-sum test, the same below; Fig. $5 \mathrm{E}$, Additional file 1: Fig. S6CD). Similarly, the core biological pathways, including immune checkpoint $(P=0.027), \mathrm{CD} 8 \mathrm{~T}$ effector $(P=0.011)$ and antigen processing machinery pathways $(P=0.027)$, were also activated in ACT-S\&HR-P patients (Additional file 1: Fig. S6EF). Additionally, analysing both immune cell infiltration and differential expression profiling revealed that the ACT-S\&HR-P subtype was enriched for both immune-activated cells and immunostimulators. For instance, immunostimulatory cells, such as M1 macrophages, NK cells and dendritic cells, showed significantly higher activity in ACT-S\&HR-P patients (Fig. 5F). However, the number of immune-suppressive cells was not elevated in this subtype (Fig. 5G). Expression profiling demonstrated that immunostimulators such as CD40, CD86 and ICOS were significantly overexpressed in this subtype (Fig. $5 \mathrm{H}$ ). The mechanisms by which ACT-S\&HR-P patients show a stronger immune response likely involve the recruitment of immune-activated cells. In particular, our results revealed that immune inhibitors, especially $I D O 1$, were significantly elevated in ACT-S\&HR-P patients (Fig. 5I). This provides a valuable reference for additional reasonable immune checkpoint blockade therapeutics for TNBC patients with the ACT-S\&HR-P subtype.

\section{Enhanced efficacy of identifying ACT responders by combining HRD and immune activation}

The above findings implied that HRD and immune cell activity might synergistically affect the ACT response; thus, we wondered whether the combination of HRD and immune activation could improve the ACT chemotherapy response. We computed the immune score (IS) of patients based on the immune response pathways (the "Methods" section) and annotated the patients with the highest $25 \%$ IS as being positive for IS (IS+). Our study showed that combined positivity (i.e. positivity for HRD, IS, or both) was significantly associated with clinical benefit $(P=1.9 \mathrm{e}-04, \log$-rank test; Fig. $6 \mathrm{~A})$ with a hazard ratio of 0.037 (95\% CI $0.0048-0.29, P=0.002$, Additional file 1: Fig. S7C) and prolonged DSS of patients $(P$ $=0.018$, Additional file 1: Fig. S7AB). More importantly, we found that the patients with combined positivity had a longer ACT failure-free interval $(P=0.013$, log-rank test; Fig. 6B). After adjusting for clinical factors, the combined positivity was found to be a significantly independent prognostic factor $(\mathrm{HR}=0.21,95 \% \mathrm{CI} 0.064$ $0.67, P=0.009$; Fig. $6 \mathrm{C}$ ). The results were consistent with the known prognostic immune markers of breast cancer (Additional file 1: Table S3, Fig. S7D-F). Incorporating IS into Cox models fit with age, tumour stage, and age and tumour stage improved the predictive accuracy of FFI $(P<0.002$, likelihood ratio test; Fig. 6D), which highlights the importance of the combination of HRD status and immune activities in ACT chemotherapy. Additionally, we found that the prognostic efficacy of combined status (AUC $=0.91$ ) was better than that of HRD status alone (AUC $=0.83$ ) or clinical factors alone $(\mathrm{AUC}=0.61)($ Fig. $6 \mathrm{E})$. These results indicated the necessity of combining HRD status with tumour immunity, which improves the efficacy of identifying ACT responders in TNBC.

To further validate those findings in an independent dataset of TNBC cases, we developed an HRD expression signature that predicts ACT response (FFI) in TCGA TNBC cohorts (the "Methods" section). We identified 15 genes that were associated with FFI, including 4 that had a better ACT response and 11 that had a worse response in HR deficiency than in HR-proficient cases (Fig. 7A, Additional file 1: Table S4). The HRD expression signature showed excellent performance in reflecting the genomic HRD status applied to TNBC patients, as demonstrated by a receiver operating characteristic (ROC) curve with an AUC of 0.89 (Fig. 7B), which was superior to other types of breast cancer samples, including all $\mathrm{BC}$ patients $(\mathrm{AUC}=0.81)$ and $\mathrm{BC}$ patients except TNBC $($ AUC $=0.77)$.

The combined score contributes to the ACT response and clinical benefit of TNBC patients

We demonstrated that the combination of the HRDrelated prognostic score (HRDPS) and IS was an effective way to predict TNBC patients who may achieve $\mathrm{pCR}$ to ACT chemotherapy, showing better prognosis in 

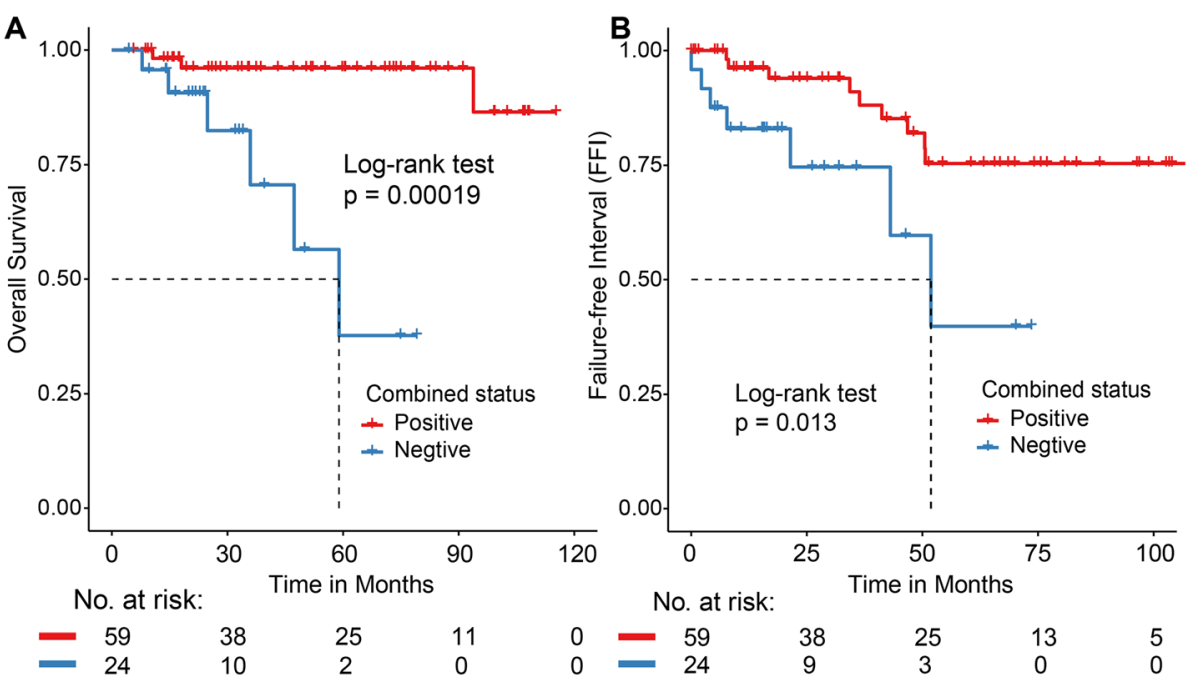

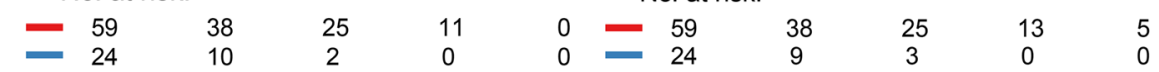

C

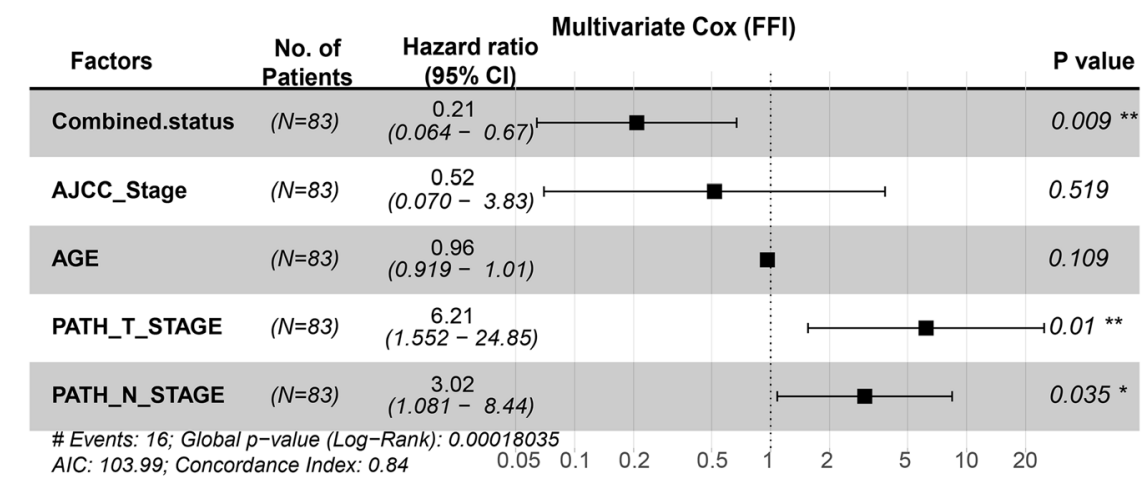

D 80
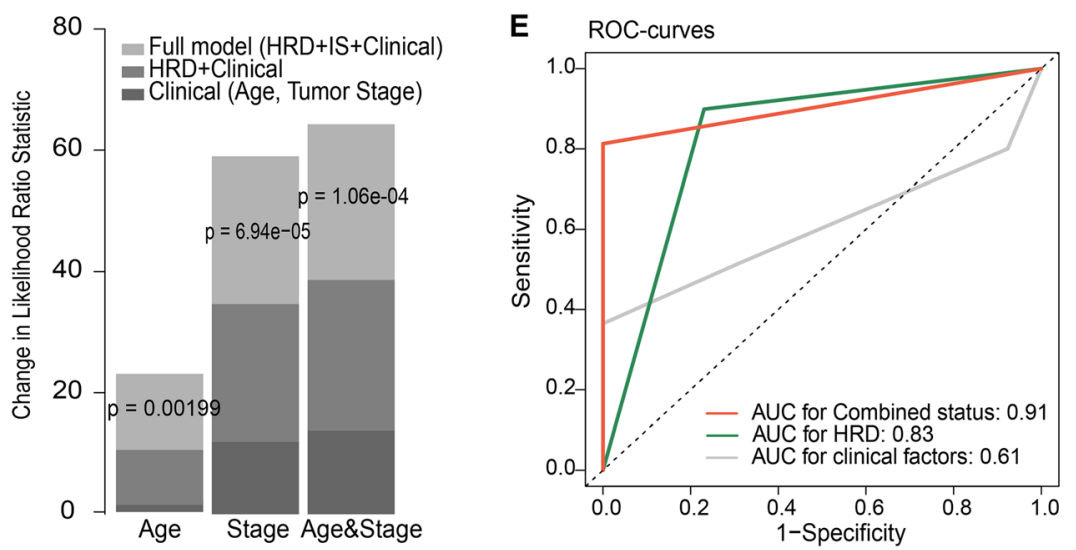

Fig. 6 Combining HRD and immune activation enhances the efficacy of identifying ACT responders. A, B OS (A) and FFI (B) by the status of combined HRD and immune activation. Log-rank test. C Multivariate Cox analysis after correcting for clinical factors such as age, AJCC stage and TNM stage. The pathology $\mathrm{M}$ stage is not included in the model due to incomplete information. ${ }^{* * *} P<0.001,{ }^{*} P<0.01,{ }^{*} P<0.05$. $\mathbf{D}$ The estimated likelihood ratio (LR) statistic of a Cox model in clinical factors, such as age, stage, and age and stage was compared. The change of LR statistic as features were added to the model was assessed for significance. $\mathbf{E}$ The area under the curve (AUC) of the combined status (orange colour), HRD status alone (green colour) and only clinical factors (grey colour) in the patient's 5-year survival time (FFI) were compared

independent validation sets (Additional file 2: Table S5) $[21,22,24]$. Our results indicated that the patients with combined positivity had longer distant relapse-free survival (DRFS; $P=3.8 \mathrm{e}-3$, log-rank test; Fig. $7 \mathrm{C}$ ). After adjusting for clinical factors, combined negativity was a significant independent risk prognostic factor in TNBC patients with a hazard ratio of 6.4 (95\% CI 2.38-17.1, $P$ $<0.001$ ) compared to combined positivity (Additional file 1: Fig. S8A). However, there was no statistical significance when using HRDPS alone, although patients with 

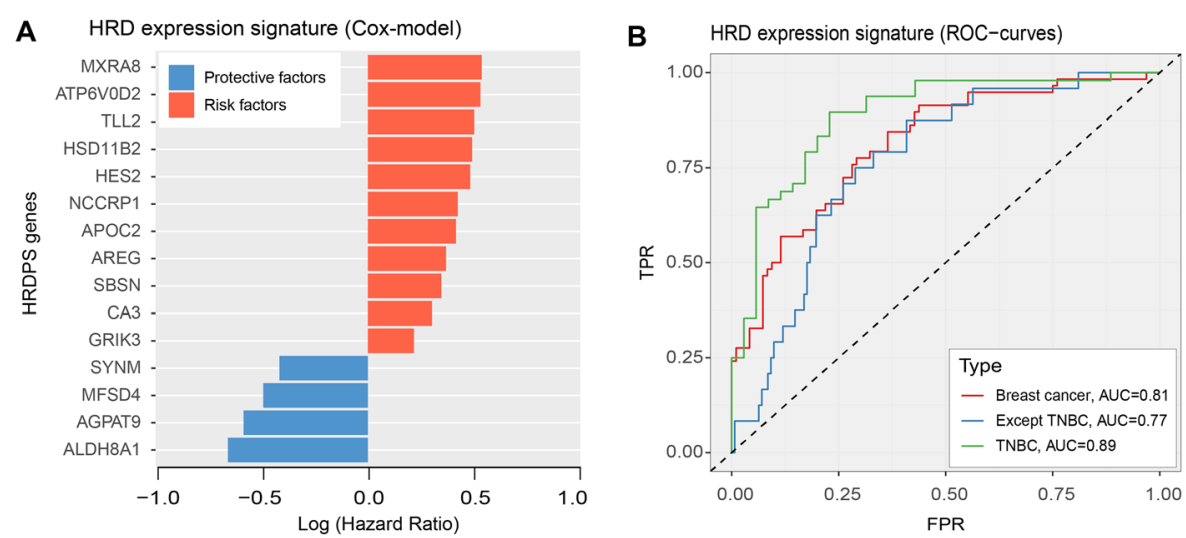

C
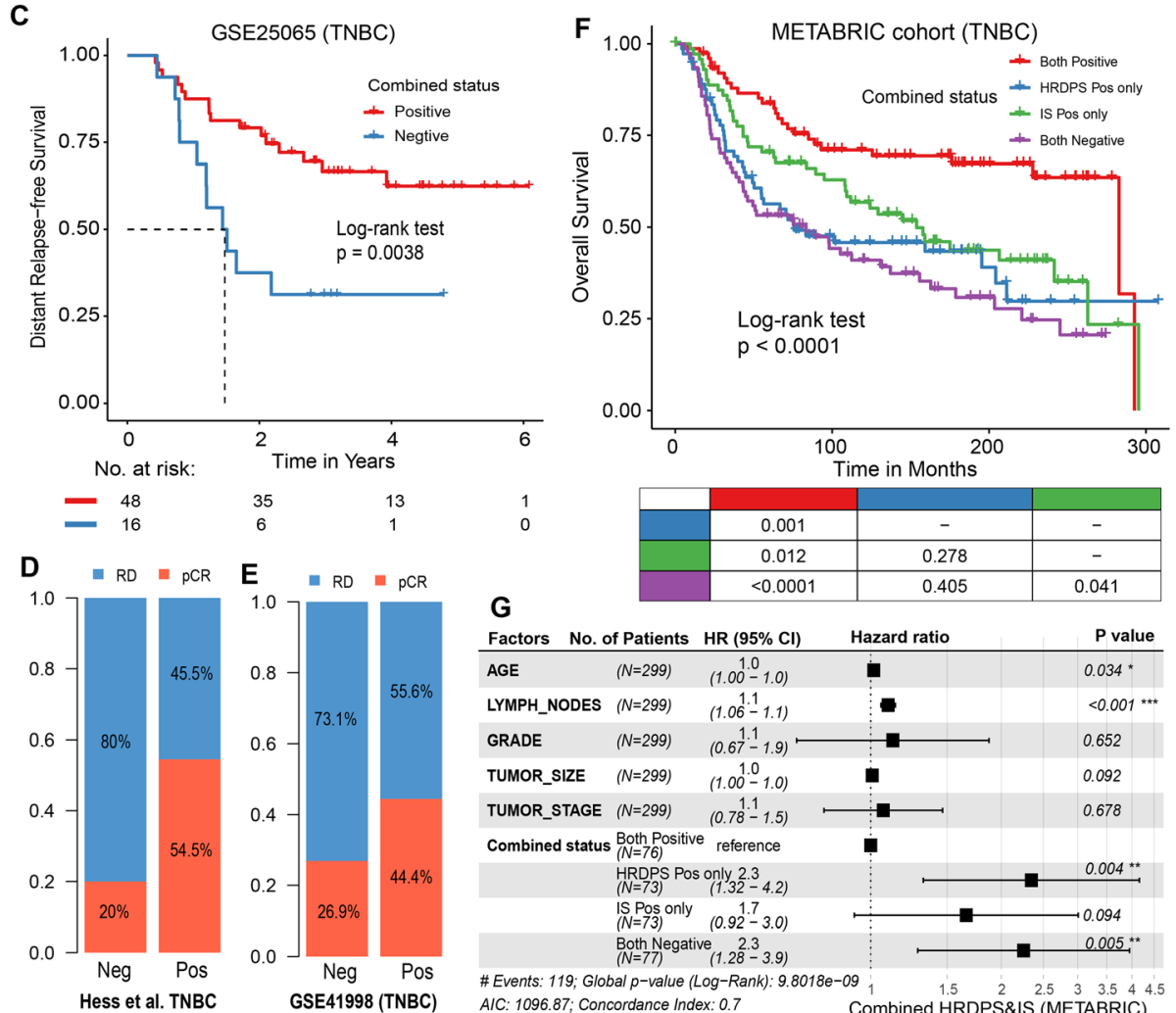

Fig. 7 Combined status contributes to ACT response and clinical prognosis of TNBC patients. A Hazard ratio of HRD expression signature was calculated using Cox regression model. Log (hazard ratio) > 0 indicates a risk factor (red); log (hazard ratio) < 0 indicates a protective factor (blue). B Performance evaluation that expression signature predicts HRD using lasso logistic regression in diverse types of breast cancer samples, including TNBC samples (green), breast cancer samples (red) and breast cancer except for TNBC (blue). C Distant relapse-free survival (DRFS) by combined status in GSE25065 TNBC cohort. D, E The bar chart shows the proportion of pCR/RD samples with the combined status in Hess et al. TNBC cohort (D) and GSE41998 TNBC cohort (E). pCR, pathological complete response; RD, residual disease. Neg, negative; Pos, positive. F OS by combined status in the METABRIC cohort. The prognostic of HRDPS alone was shown in Additional file 1: Fig. S8C. G The forest plot shows the combined status as an independent prognostic factor for TNBC patients using multivariate Cox regression analysis (METABRIC cohort)

HRD-positive had better DRFS $(P=0.062, \log$-rank test; Additional file 1: Fig. S8B). In addition, the patients with combined positivity had higher pCR rates of ACT in two independent validation sets $(54.5 \%$ for Hess et al. TNBC, 44.4\% for GSE1998 TNBC) compared with combined negativity cases $(20 \%$ for Hess et al. TNBC, $26.9 \%$ for GSE1998 TNBC; Fig. 7D, E).
Furthermore, we analysed the impact of HRDPS, IS and combined status on the prognosis of TNBC patients who were treated with chemotherapy [23]. The results showed that the combination of HRD status and tumour immune activation showed a strong correlation with patient OS $(P<0.0001$, log-rank test; Fig. $7 \mathrm{~F})$. The patients with positivity for both factors showed the longest OS 
compared with those with other statuses $(P<0.0001$ compared with both negative, $P=0.001$ compared with HRDPS-positive only and $P=0.012$ compared with ISpositive only, log-rank test; Fig. 7F). Multivariate Cox regression showed that the patients who were negative for both $(\mathrm{HR}=2.395 \%$ CI 1.26-3.9, $P=0.005)$ and HRDP S-positive only $(\mathrm{HR}=2.395 \% \mathrm{CI} 1.32-4.2, P=0.004)$ showed a significantly worse prognosis compared with patients who were positive for both (Fig. 7G).

Similarly, we acquired consistent results in two additional validation sets of TNBC patients who received ACT intervention [22, 25]. For example, the patients with positivity for both HRDPS and IS showed the longest DRFS (GSE25055; $P=0.007$ compared with ISpositive only; $P=0.041$ compared with HRDPS-positive only; $P=0.053$ compared with both negative, log-rank test; Additional file 1: Fig. S9A) and the best survival outcomes for DSS (Chin et al.; $P=0.037$ compared with HRDPS-positive only; $P=0.049$ compared with both negative, log-rank test; Additional file 1: Fig. S9B). These results demonstrated that the combination of HRD and tumour immune activation can indeed contribute to the ACT chemotherapy response and clinical outcomes of TNBC patients.

\section{Discussion}

In this study, we developed an integrated strategy to predict the ACT chemotherapy response through the combination of HR deficiency and immune activation in TNBC patients. Our method integrated multiomics data to ensure that we can not only characterize the HRD phenotype of TNBC using different methods but also analyse the effect on the ACT chemotherapy response in combination with the immune microenvironment. The presence of $B R C A 1 / 2$ mutations or tumour genomic instability (HRD score $\geq 42$ ) is surrogate markers of HR deficiency $[8,10]$, and a positive immune score is a surrogate of interferon-primed immune checkpoints in the tumour microenvironment [49]. The presence of one or both tumour features was associated with longer FFI $(\mathrm{HR}=0.21, P=0.009)$ and $\mathrm{OS}(\mathrm{HR}=0.037, P=0.002)$ in TNBC patients. A transcriptional HRD signature related to the ACT response was independently validated to be significantly associated with improved survival in the GEO cohort $(P=0.0038)$ and the METABRIC dataset $(P<0.0001)$ and increased ACT pCR rates of TNBC patients. These results are clinically relevant and suggest that combining HRD status and tumour immunity may aid in the selection of TNBC patients who would benefit from ACT chemotherapy.

TNBC is a breast cancer subtype with fairly abundant defects in DNA damage repair, especially HRD (approximately $67 \%$ ) $[8,10]$, which encourages us to start from the perspective of DNA DSBs and develop treatment strategies that are sensitive to DNA damage inducers or DNA synthesis inhibitors $[9,10]$. Mutations in BRCA1/2 genes, especially germline variations, along with other Fanconi anaemia (FA) pathway genes (such as $N B N$, RAD54L, ATM), are prototypic molecular alterations that confer HRD in breast cancer [8]. Unfortunately, we cannot acquire information about germline BRCA1/2 variation from public data resources. The HRD score is an algorithmic assessment of three measures of tumour genomic instability, namely, loss of heterozygosity, telomeric allelic imbalance and large-scale state transitions, which is a recognized indicator to characterize HRDs $[10,40]$. BRCA1 promoter hypermethylation, as an inactivator of epigenetic modifications, was associated with a gene expression profile similar to that of inherited BRCA1 mutation-associated breast cancer [9, 41]. Mutational signature 3 is a genomic feature associated with failure of DNA DSB repair in breast cancer and is highly related to $B R C A 1 / 2$ variation [41]. We identified HRdeficient patients with $B R C A 1 / 2$ mutations or HRD

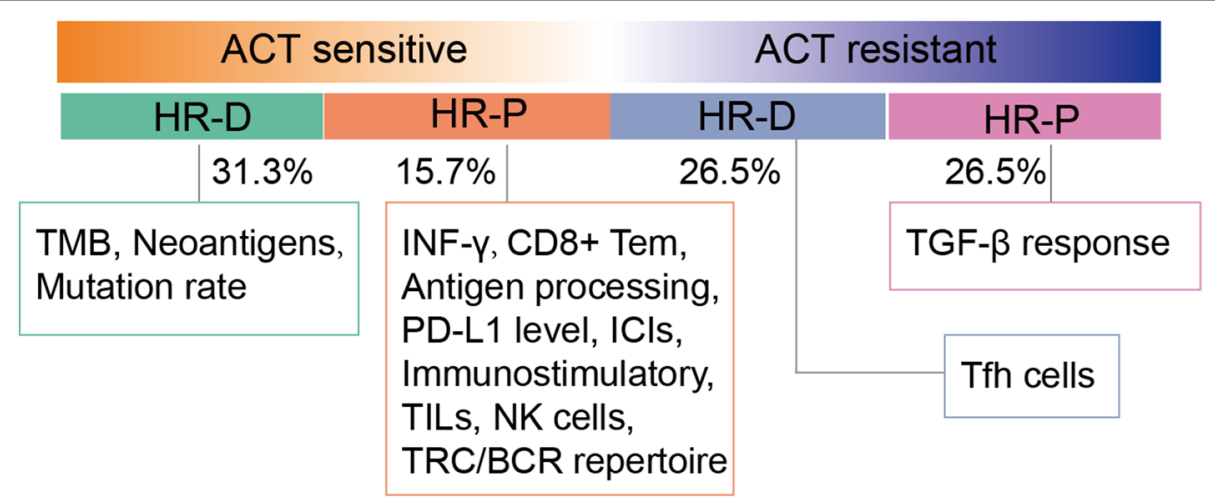

Fig. 8 The characteristics summary of TNBC patients with HRD and ACT response. TMB, tumour mutation burden; ICls, immune checkpoint inhibitors; TILs, tumour-infiltrating lymphocytes; NK cells, natural killer cells; TCR/BCR, T cell/B cell receptor; Tfh cells, T follicular helper cells 
scores $\geq 42$ and indeed found that HR deficiency was associated with higher SBS3 activity, HRR gene mutations and $B R C A 1$ promoter hypermethylation.

In agreement with previous observations, we found that HR deficiency was associated with better survival of TNBCs [8]. In addition, we further determined the FFI for TNBC patients who received ACT chemotherapy based on the period from the end of treatment to tumour progression/recurrence or death and found that HR deficiency was related to the patient durable response to ACT chemotherapy $(P=0.046)$. In cases of incomplete therapy response information from public databases, we explicitly classified TNBC patients as ACT-sensitive or ACT-resistant according to FFI and whether the tumour had progressed or complete responses after ACT treatment. This allowed us to further characterize the correlation between HRD status and ACT response. Considering the results of previous studies, the identification of TNBC patients who are sensitive to ACT is limited if only HRD status was considered [8]. The combined analysis of HRD status and the immune microenvironment provided us with an additional reference (Fig. 8), which made up for the limitations. Through transcriptional HRD signature analysis, we indicated that the HRD gene expression score was better at prognosis when combined with the immune score than their genetically determined HRD status. Our results demonstrated that the immunostimulatory cells and immune inhibitors (such as PD-L1, CTLA-4 and IDO1) were significantly elevated in ACT-S\&HR-P patients, providing additional rationale for the use of immune checkpoint blockade as a therapeutic approach.

The relative proportions of infiltrating immune cells were inferred based on gene expression profiles. However, mRNA only-based assessment of the immune infiltrate meets with several limitations. The architectural pattern and spatial distribution of TILs might be not captured by tumour transcriptomes. In addition, the differences of cellular RNA content may lead to estimation biases. And the calculation method particularly depends on the robustness of TIL marker genes [50, 51]. Therefore, it is necessary to consider the combination of immunohistochemistry to assess the immune infiltrate in future studies.

\section{Conclusions}

In summary, our findings highlighted that HR deficiency could prolong ACT chemotherapy benefits (failure-free interval) and predicted an intensified ACT response in TNBC patients by combining HRD and immune activation. The combination synergistically contributes to the clinical outcomes of TNBC patients and enhances the efficacy of identifying ACT chemotherapy responders, which resolves the issue that ACT chemotherapy- sensitive patients cannot be clearly identified using HRD status alone. The combined status of HRD and immune activation based on gene expression assay can be used as a potential prognostic marker for TNBC patients, which suggests that combining the two types of characteristics has important application value in guiding the use of ACT chemotherapy in TNBCs. This provides a molecular basis for accurately identifying ACT chemotherapy responders and has prospective significance in clinical trials.

\section{Abbreviations}

ACT: Anthracycline, cyclophosphamide and taxane; AUC: Area under the curve; Cls: Confidence intervals; DEGs: Differentially expressed genes;

DSBs: Double-strand breaks; DSS: Disease-specific survival; ER: Oestrogen receptors; FDR: False discovery rate; FFI: Failure-free interval; GEO: Gene Expression Omnibus; GSEA: Gene set enrichment analysis; HER2: Human epidermal growth factor receptor 2; HRD: Homologous recombination repair deficiency; HRDPS: HRD-related prognostic score; ICB: Immune checkpoint blockade; ICIs: Immune checkpoint inhibitors; IHC: Immunohistochemistry; IS: Immune score; LOH: Loss of heterozygosity; LST: Large-scale transitions; METABRIC: Molecular Taxonomy of Breast Cancer International Consortium; ntAl: Telomeric allelic imbalances; OS: Overall survival; pCR: Pathological complete response; PR: Progesterone receptors; PS: Prognostic score; RD: Residual disease; ROC: Receiver operating characteristic; TCGA: The Cancer Genome Atlas; TLI: Tumour lymphocyte infiltration; TMB: Tumour mutation burden; TNBC: Triple-negative breast cancer; TSS: Transcription start site; WES: Whole-exome sequencing

\section{Supplementary Information}

The online version contains supplementary material available at https://doi. org/10.1186/s12916-021-02068-4.

\section{Additional file 1: Figure S1. The mutations of homologous recombination repair genes in patients. Figure S2. Homologous recombination repair defects correlate with clinical benefits. Figure S3. Immune infiltration level of TNBC patients. Figure S4. Analysis of the immune microenvironment mechanism of TNBC patients. Figure S5. Representative gene set enrichment analysis plot. Figure S6. Immune checkpoints activated in the ACT-S\&HR-P subtype. Figure S7. Combining HRD and immune checkpoints correlates with clinical benefits. Figure S8. HRD status and prognosis of TNBC patients. Figure S9. Combined status contributes to prognosis of TNBC patients. Table S1. Basic data in- formation of TNBC patients. Table S2. Immune activation-related path- ways and their genes. Table S3. Immune markers related to breast cancer. Table S4. Cox model results of HRD expression signature. \\ Additional file 2: Table S5. The values for HRD score (transcriptional HRD signature) and immune score of TNBC patients.}

\section{Acknowledgements}

We would like to thank the staff members of TCGA Research Network, the CBioPortal, the GEO data, the UCSC Xena data portal and the CIBERSORT portal, as well as all the authors for making their valuable research data public.

\section{Authors' contributions}

Study concept and design: Liao. Acquisition, analysis or interpretation of the data: Liao and Jiang. Drafting of the manuscript: Liao, Jiang and Yang. Critical revision of the manuscript for important intellectual content: all authors. Study supervision: Li and Xiao. All authors read and approved the final manuscript.

\section{Funding}

This work was supported by the National Key R\&D Program of China (Grant No. 2018YFC2000100), the National Natural Science Foundation of China (Grant No. 61873075, 32070673, 31871336), the Heilongjiang Provincial 
Natural Science Foundation (Grant No. YQ2019C012), the Heilongjiang Postdoctoral Foundation (Grant No. LBH-Q18099) and the Program for Young Scholars with Creative Talents in Heilongjiang Province (UNPYSCT-2017059).

\section{Availability of data and materials}

All of the data we used in this study were publicly available as described in the "Methods" section

\section{Declarations}

\section{Ethics approval and consent to participate}

This research has been conducted using publicly available datasets. Deidentified data were used, and no ethical approval was required.

\section{Consent for publication}

Not applicable.

\section{Competing interests}

The authors declare that they have no competing interests.

\section{Author details}

'College of Bioinformatics Science and Technology, Harbin Medical University, Harbin 150081, Heilongjiang, China. ${ }^{2}$ Department of Ultrasonic Medicine, The First Affiliated Hospital of Harbin Medical University, Harbin 150010, Heilongjiang, China. ${ }^{3}$ Key Laboratory of University in Heilongjiang Province, Department of Pharmacy, The Second Affiliated Hospital of Harbin Medical University, Harbin 150086, China. ${ }^{4}$ Key Laboratory of Cardiovascular Medicine Research, Harbin Medical University, Ministry of Education, Harbin 150081, Heilongjiang, China.

\section{Received: 8 May 2021 Accepted: 20 July 2021}

\section{Published online: 01 September 2021}

\section{References}

1. Torre LA, Bray F, Siegel RL, Ferlay J, Lortet-Tieulent J, Jemal A. Global cancer statistics, 2012. CA. 2015;65(2):87-108. https://doi.org/10.3322/caac.21262.

2. Yin L, Duan JJ, Bian XW, Yu SC. Triple-negative breast cancer molecular subtyping and treatment progress. Breast Cancer Res. 2020;22(1):61.

3. Gianni L, Mansutti M, Anton A, Calvo L, Bisagni G, Bermejo B, et al. Comparing neoadjuvant nab-paclitaxel vs paclitaxel both followed by anthracycline regimens in women with ERBB2/HER2-negative breast cancerthe Evaluating Treatment with Neoadjuvant Abraxane (ETNA) trial: a randomized phase 3 clinical trial. JAMA Oncol. 2018;4(3):302-8. https://doi. org/10.1001/jamaoncol.2017.4612.

4. Gradishar WJ, Anderson BO, Abraham J, Aft R, Agnese D, Allison KH, et al. Breast Cancer, version 3.2020, NCCN Clinical Practice Guidelines in Oncology. J Natl Compr Cancer Netw. 2020:18(4):452-78.

5. Budd GT, Barlow WE, Moore HC, Hobday TJ, Stewart JA, Isaacs C, et al. SWOG S0221: a phase III trial comparing chemotherapy schedules in highrisk early-stage breast cancer. J Clin Oncol. 2015;33(1):58-64.

6. Wright WD, Shah SS, Heyer WD. Homologous recombination and the repair of DNA double-strand breaks. J Biol Chem. 2018;293(27):10524-35. https:// doi.org/10.1074/jbc.TM118.000372.

7. Tutt A, Robson M, Garber JE, Domchek SM, Audeh MW, Weitzel JN, et al. Oral poly(ADP-ribose) polymerase inhibitor olaparib in patients with BRCA1 or BRCA2 mutations and advanced breast cancer: a proof-of-concept trial. Lancet. 2010;376(9737):235-44. https://doi.org/10.1016/S0140-6736(1 0)60892-6.

8. Sharma P, Barlow WE, Godwin AK, Pathak H, Isakova K, Williams D, et al. Impact of homologous recombination deficiency biomarkers on outcomes in patients with triple-negative breast cancer treated with adjuvant doxorubicin and cyclophosphamide (SWOG S9313). Ann Oncol. 2018;29(3): 654-60.

9. Hoppe MM, Sundar R, Tan DSP, Jeyasekharan AD. Biomarkers for homologous recombination deficiency in cancer. J Natl Cancer Inst. 2018; 110(7):704-13. https://doi.org/10.1093/jnci/djy085.

10. Telli ML, Timms KM, Reid J, Hennessy B, Mills GB, Jensen KC, et al. Homologous recombination deficiency (HRD) score predicts response to platinum-containing neoadjuvant chemotherapy in patients with triplenegative breast cancer. Clin Cancer Res. 2016;22(15):3764-73.
11. Pohl-Rescigno E, Hauke J, Loibl S, Mobus V, Denkert C, Fasching PA, et al. Association of germline variant status with therapy response in high-risk early-stage breast cancer: a secondary analysis of the GeparOcto Randomized Clinical Trial. JAMA Oncol. 2020;6(5):744-8. https://doi.org/10.1 001/jamaoncol.2020.0007.

12. Mittendorf EA, Zhang H, Barrios $\mathrm{CH}$, Saji S, Jung $\mathrm{KH}$, Hegg $\mathrm{R}$, et al. Neoadjuvant atezolizumab in combination with sequential nab-paclitaxel and anthracycline-based chemotherapy versus placebo and chemotherapy in patients with early-stage triple-negative breast cancer (IMpassion031): a randomised, double-blind, phase 3 trial. Lancet. 2020;396(10257):1090-100 https://doi.org/10.1016/S0140-6736(20)31953-X.

13. Untch M, Jackisch C, Schneeweiss A, Conrad B, Aktas B, Denkert C, et al. Nab-paclitaxel versus solvent-based paclitaxel in neoadjuvant chemotherapy for early breast cancer (GeparSepto-GBG 69): a randomised, phase 3 trial. Lancet Oncol. 2016;17(3):345-56. https://doi.org/10.1016/S1470-2045(1 5)00542-2.

14. Sato $H$, Niimi A, Yasuhara T, Permata TBM, Hagiwara Y, Isono M, et al. DNA double-strand break repair pathway regulates PD-L1 expression in cancer cells. Nat Commun. 2017;8(1):1751. https://doi.org/10.1038/s41467-017-01 883-9.

15. Stewart RA, Pilie PG, Yap TA. Development of PARP and immunecheckpoint inhibitor combinations. Cancer Res. 2018;78(24):6717-25. https:// doi.org/10.1158/0008-5472.CAN-18-2652.

16. Mouw KW, Goldberg MS, Konstantinopoulos PA, D'Andrea AD. DNA damage and repair biomarkers of immunotherapy response. Cancer Discov. 2017;7(7):675-93. https://doi.org/10.1158/2159-8290.CD-17-0226.

17. Voorwerk L, Slagter M, Horlings HM, Sikorska K, van de Vijver KK, de Maaker $\mathrm{M}$, et al. Immune induction strategies in metastatic triple-negative breast cancer to enhance the sensitivity to PD-1 blockade: the TONIC trial. Nat Med. 2019;25(6):920-8. https://doi.org/10.1038/s41591-019-0432-4.

18. Scurr M, Pembroke T, Bloom A, Roberts D, Thomson A, Smart K, et al. Lowdose cyclophosphamide induces antitumor T-cell responses, which associate with survival in metastatic colorectal cancer. Clin Cancer Res. 2017; 23(22):6771-80.

19. Berger AC, Korkut A, Kanchi RS, Hegde AM, Lenoir W, Liu W, et al. A comprehensive pan-cancer molecular study of gynecologic and breast cancers. Cancer Cell. 2018;33(4):690-705 e699. https://doi.org/10.1016/j. ccell.2018.03.014.

20. Wishart DS, Knox C, Guo AC, Cheng D, Shrivastava S, Tzur D, et al. DrugBank: a knowledgebase for drugs, drug actions and drug targets. Nucleic Acids Res. 2008;36(Database issue):D901-6. https://doi.org/10.1093/ nar/gkm958.

21. Hatzis C, Pusztai L, Valero V, Booser DJ, Esserman L, Lluch A, et al. A genomic predictor of response and survival following taxane-anthracycline chemotherapy for invasive breast cancer. JAMA. 2011:305(18):1873-81. https://doi.org/10.1001/jama.2011.593.

22. Horak CE, Pusztai L, Xing G, Trifan OC, Saura C, Tseng LM, et al. Biomarker analysis of neoadjuvant doxorubicin/cyclophosphamide followed by ixabepilone or paclitaxel in early-stage breast cancer. Clin Cancer Res. 2013; 19(6):1587-95.

23. Curtis C, Shah SP, Chin SF, Turashvili G, Rueda OM, Dunning MJ, et al. The genomic and transcriptomic architecture of 2,000 breast tumours reveals novel subgroups. Nature. 2012;486(7403):346-52. https://doi.org/10.1038/na ture10983.

24. Hess KR, Anderson K, Symmans WF, Valero V, Ibrahim N, Mejia JA, et al. Pharmacogenomic predictor of sensitivity to preoperative chemotherapy with paclitaxel and fluorouracil, doxorubicin, and cyclophosphamide in breast cancer. J Clin Oncol. 2006;24(26):4236-44.

25. Chin K, DeVries S, Fridlyand J, Spellman PT, Roydasgupta R, Kuo WL, et al. Genomic and transcriptional aberrations linked to breast cancer pathophysiologies. Cancer Cell. 2006;10(6):529-41. https://doi.org/10.1016/j.ccr.2006.10.009.

26. Thorsson V, Gibbs DL, Brown SD, Wolf D, Bortone DS, Ou Yang TH, et al. The immune landscape of cancer. Immunity. 2018;48(4):812-30 e814. https://doi.org/10.1016/j.immuni.2018.03.023.

27. Rosenthal R, McGranahan N, Herrero J, Taylor BS, Swanton C. DeconstructSigs: delineating mutational processes in single tumors distinguishes DNA repair deficiencies and patterns of carcinoma evolution. Genome Biol. 2016;17(1):31. https://doi.org/10.1186/s13059-016-0893-4.

28. Davies H, Glodzik D, Morganella S, Yates LR, Staaf J, Zou X, et al. HRDetect is a predictor of BRCA1 and BRCA2 deficiency based on mutational signatures. Nat Med. 2017;23(4):517-25. https://doi.org/10.1038/nm.4292. 
29. Sztupinszki Z, Diossy M, Krzystanek M, Borcsok J, Pomerantz MM, Tisza V et al. Detection of molecular signatures of homologous recombination deficiency in prostate cancer with or without BRCA1/2 mutations. Clin Cancer Res. 2020;26(11):2673-80.

30. Newman AM, Steen CB, Liu CL, Gentles AJ, Chaudhuri AA, Scherer F, et al. Determining cell type abundance and expression from bulk tissues with digital cytometry. Nat Biotechnol. 2019;37(7):773-82. https://doi.org/10.1038/ s41587-019-0114-2.

31. Jiang P, Gu S, Pan D, Fu J, Sahu A, Hu X, et al. Signatures of T cell dysfunction and exclusion predict cancer immunotherapy response. Nat Med. 2018;24(10):1550-8. https://doi.org/10.1038/s41591-018-0136-1.

32. Subramanian A, Tamayo P, Mootha VK, Mukherjee S, Ebert BL, Gillette MA, et al. Gene set enrichment analysis: a knowledge-based approach for interpreting genome-wide expression profiles. Proc Natl Acad Sci U S A. 2005;102(43):15545-50. https://doi.org/10.1073/pnas.0506580102.

33. Aran D, Hu Z, Butte AJ. xCell: digitally portraying the tissue cellular heterogeneity landscape. Genome Biol. 2017;18(1):220. https://doi.org/10.11 86/s13059-017-1349-1.

34. Mariathasan S, Turley SJ, Nickles D, Castiglioni A, Yuen K, Wang Y, et al. TGF $\beta$ attenuates tumour response to PD-L1 blockade by contributing to exclusion of T cells. Nature. 2018;554(7693):544-8. https://doi.org/10.1038/na ture25501.

35. Angelova M, Charoentong P, Hackl H, Fischer ML, Snajder R, Krogsdam AM, et al. Characterization of the immunophenotypes and antigenomes of colorectal cancers reveals distinct tumor escape mechanisms and novel targets for immunotherapy. Genome Biol. 2015;16(1):64. https://doi.org/1 0.1186/s13059-015-0620-6.

36. Jiang YZ, Ma D, Suo C, Shi J, Xue M, Hu X, et al. Genomic and transcriptomic landscape of triple-negative breast cancers: subtypes and treatment strategies. Cancer Cell. 2019;35(3):428-40 e425. https://doi.org/1 0.1016/j.ccell.2019.02.001.

37. Yang B, Chou J, Tao Y, Wu D, Wu X, Li X, et al. An assessment of prognostic immunity markers in breast cancer. NPJ Breast Cancer. 2018;4(1):35. https:// doi.org/10.1038/s41523-018-0088-0

38. Hubalek M, Czech T, Muller H. Biological subtypes of triple-negative breast cancer. Breast Care. 2017;12(1):8-14. https://doi.org/10.1159/000455820.

39. Hoadley KA, Yau C, Hinoue T, Wolf DM, Lazar AJ, Drill E, et al. Cell-of-origin patterns dominate the molecular classification of 10,000 tumors from 33 types of cancer. Cell. 2018;173(2):291-304 e296. https://doi.org/10.1016/j. cell.2018.03.022.

40. Marquard AM, Eklund AC, Joshi T, Krzystanek M, Favero F, Wang ZC, et al. Pan-cancer analysis of genomic scar signatures associated with homologous recombination deficiency suggests novel indications for existing cancer drugs. Biomarker Res. 2015;3(1):9. https://doi.org/10.1186/s40364-015-0033-4.

41. Polak P, Kim J, Braunstein LZ, Karlic R, Haradhavala NJ, Tiao G, et al. A mutational signature reveals alterations underlying deficient homologous recombination repair in breast cancer. Nat Genet. 2017;49(10):1476-86. https://doi.org/10.1038/ng.3934

42. Staaf J, Glodzik D, Bosch A, Vallon-Christersson J, Reutersward C, Hakkinen J, et al. Whole-genome sequencing of triple-negative breast cancers in a population-based clinical study. Nat Med. 2019;25(10):1526-33. https://doi. org/10.1038/s41591-019-0582-4.

43. Knijnenburg TA, Wang L, Zimmermann MT, Chambwe N, Gao GF, Cherniack $A D$, et al. Genomic and molecular landscape of DNA damage repair deficiency across The Cancer Genome Atlas. Cell Rep. 2018;23(1):239-54 e236. https://doi.org/10.1016/j.celrep.2018.03.076.

44. Sewell AK. Why must T cells be cross-reactive? Nat Rev Immunol. 2012;12(9): 669-77. https://doi.org/10.1038/nri3279.

45. Smith-Garvin JE, Koretzky GA, Jordan MS. T cell activation. Annu Rev Immunol. 2009;27(1):591-619. https://doi.org/10.1146/annurev.immunol.021 908.132706 .

46. Gubin MM, Zhang $X$, Schuster $H$, Caron E, Ward JP, Noguchi T, et al. Checkpoint blockade cancer immunotherapy targets tumour-specific mutant antigens. Nature. 2014;515(7528):577-81. https://doi.org/10.1038/na ture13988.

47. Yadav M, Jhunjhunwala S, Phung QT, Lupardus P, Tanguay J, Bumbaca S, et al. Predicting immunogenic tumour mutations by combining mass spectrometry and exome sequencing. Nature. 2014;515(7528):572-6. https:// doi.org/10.1038/nature14001.

48. Arora S, Velichinskii R, Lesh RW, Ali U, Kubiak M, Bansal P, et al. Existing and emerging biomarkers for immune checkpoint immunotherapy in solid tumors. Adv Therapy. 2019;36(10):2638-78. https://doi.org/10.1007/s12325019-01051-z.

49. Farkkila A, Gulhan DC, Casado J, Jacobson CA, Nguyen H, Kochupurakkal B, et al. Immunogenomic profiling determines responses to combined PARP and PD-1 inhibition in ovarian cancer. Nat Commun. 2020;11(1):1459. https://doi.org/10.1038/s41467-020-15315-8.

50. Newman AM, Alizadeh AA. High-throughput genomic profiling of tumorinfiltrating leukocytes. Curr Opin Immunol. 2016;41:77-84. https://doi.org/1 0.1016/j.coi.2016.06.006.

51. Hackl H, Charoentong P, Finotello F, Trajanoski Z. Computational genomics tools for dissecting tumour-immune cell interactions. Nat Rev Genet. 2016; 17(8):441-58. https://doi.org/10.1038/nrg.2016.67.

\section{Publisher's Note}

Springer Nature remains neutral with regard to jurisdictional claims in published maps and institutional affiliations.
Ready to submit your research? Choose BMC and benefit from:

- fast, convenient online submission

- thorough peer review by experienced researchers in your field

- rapid publication on acceptance

- support for research data, including large and complex data types

- gold Open Access which fosters wider collaboration and increased citations

- maximum visibility for your research: over $100 \mathrm{M}$ website views per year

At BMC, research is always in progress.

Learn more biomedcentral.com/submissions 